\title{
GMSD-based Perceptually Motivated Non-local Means Filter for Image Denoising
}

\author{
Mohtashim Baqar*, Sian Lun Lau* and Mansoor Ebrahim ${ }^{\dagger}$ \\ Department of Computing and Information Systems, \\ School of Science and Technology, \\ Sunway University, Malaysia* \\ Iqra University (Main Campus), Karachi, Pakistan ${ }^{\dagger}$ \\ mohtash.b@imail.sunway.edu.my, sianlunl@sunway.edu.my,mebrahim@iqra.edu.pk
}

\begin{abstract}
Due to increasing proliferation of multimedia signals, specifically, image, video and their applications in our daily life, it is indispensable to have methods that can efficiently predict and correct visual quality of images with high measures of accuracy. Therefore, in this work a state-of-theart (STOA) image quality assessment (IQA) metric, gradient magnitude similarity deviation (GMSD) has been incorporated in a STOA least-square-based non-local means (NLM) filtering framework for image denoising. The denoising process works by estimating and weighting neighbouring patches similar to the patch being denoised in terms of Euclidean distance (ED) and GMSD coefficient. The overall process is broken down into two steps; initially, local noise estimates for the underlying noisy patch are approximated and removed, then the refined patch is fed to the weighting process as the final step. Further, the proposed methodology also helps in mitigating the patch jittering blur effect (PJBE) and over smoothing of denoised images as observed with conventional NLM algorithm. Experimental evaluations based on visual-quality assessment and least-squarebased metrics have shown that the proposed algorithm yields better denoised image estimates than the conventional NLM algorithm. Moreover, experiments conducted on a subjective database, i.e. CSIQ, have shown higher performance in terms of peak signal-to-noise ratio (PSNR), structural similarity (SSIM) and GMSD coefficients. The resultant denoised images were in high correlation with the subjective judgements compared to the ones obtained with conventional NLM algorithm.
\end{abstract}

Index Terms-Image quality assessment, perceptual image filtering, non-local means filter, image denoising, visual perception.

\section{INTRODUCTION}

Least-square-based mechanisms are used extensively in multimedia (image, video and speech) signal processing. However, they often prove to be poor measures of visual quality [1]. Many multimedia applications have the human vision as the ultimate receptor, because it is imperative to produce highquality visuals that are compatible with human perception. The most popular, straightforward and computationally efficient quality assessment metrics are the mean square error (MSE) and peak signal-to-noise ratio (PSNR). However, the visual quality is not guaranteed because both look for visible errors for quality assessment. Even a higher PSNR value may lead to poor perceived visual quality.

Since early 2000, numerous perceptual quality assessment metrics have been developed for effective IQA. Though, an initial breakthrough was made with structural similarity (SSIM) index [1]. It was one of the most popular similarity measure, which has shown to outperform MSE and PSNR in effectively predicting perceived image quality. Since then, SSIM and its variants have been used in wide variety of visual computing applications, including, image compression, image restoration and pattern recognition. After SSIM, different stateof-the-art (STOA) IQA algorithms have been developed; with each utilizing different aspects and characteristics of an image for effective quality assessment. Among them is GMSD, a STOA IQA algorithm, that deals with almost every type of distortion effectively and is computationally efficient as well. Instead of just utilizing GMSD for image quality assessment and evaluation of algorithms, researchers have also started using it in the design of different image processing systems and algorithms as done in [2].

In this work, we have used GMSD for image denoising. Though a great deal of work has already been carried out to solve this practical problem, most of the existing frameworks use least-square as the primary metric. One of the STOA and most famous image denoising algorithm is the non-local means (NLM) filter [3]. NLM filtering utilizes a weighted averaging technique. The term non-local implies that the search of similar pixels or patches would not just be confined to a local neighbourhood of these pixels or patches for denoising, but a broader search space. Moreover, it utilizes MSE as the metric to find similar pixels or patches with the one to be denoised. The denoising process works well but still can't guarantee visual quality. Hence, this serves as a motivation to incorporate a perceptual metric in the denoising framework. Authors in [4] incorporated SSIM into NLM framework to replace MSE with success, but SSIM itself is computationally expensive and requires tuning as well. Furthermore, results in [5] have proved the dominance of GMSD over SSIM in terms of accuracy and computational efficiency. So, this motivated us to use GMSD as the metric to build a perceptually and computationally efficient denoising framework.

In our proposed scheme, we will not completely remove MSE from the NLM framework. Instead, we use it as a weighting coefficient along with GMSD. Both the MSE and GMSD metrics complement each other in the estimation of weighting coefficients. Moreover, the most significant advan- 
tage that GMSD holds is its robustness. It enables the capture of structures in an image well, even at higher noise levels. The proposed framework is a two-step approach; Firstly, local noise estimates for the underlying patch are approximated. Secondly, the GMSD-based similarity and weighting process are applied.

The rest of the paper is organized as follows: In section II, a detailed description of NLM and the proposed algorithm is provided. In addition, complete algorithmic details of the proposed model are also included in this section. Furthermore, the experimental specifications and results along with details of database and performance evaluation measures are presented and discussed in section III. Finally, the work is concluded in section IV.

\section{Problem And Proposed Methodology}

In this section, details of the NLM algorithm [6] and the proposed GMSD-based NLM algorithm are briefly discussed.

\section{A. Problem Formulation}

NLM algorithm works on the principle of replacing each pixel in the noisy image with a weighted average of all the pixels having similar attributes (intensity) in a broader but confined search space. Mathematically, an NLM filter is defined as in Equation 1.

$$
U_{i}^{(N L M)}=\sum_{j \in \Omega} \omega(i, j) g(j)
$$

where, $g(j)$ represents the centre pixel of a patch of radius $\Omega$ in the noisy image. Moreover, this patch is weighted depending upon its similarity with the underlying noisy patch using the factor $\omega(i, j)$. Furthermore, $\omega(i, j)$ is the weighting factor between the patch to be denoised and a patch in the search space as defined in equation 2 . The weighted average between two corresponding patches, $g\left(N_{i}\right)$ and $g\left(N_{j}\right)$, is calculated using Euclidean distance (ED).

$$
\omega(i, j)=\frac{1}{Z(i)} e^{-\frac{\left\|g\left(N_{j}\right)-g\left(N_{i}\right)\right\|_{2}^{2}}{h^{2}}}
$$

where, $Z(i)$ is the normalization factor in the weighting function $w(i, j)$ and $h$ is the filtering parameter, whose value depends upon the magnitude of noise intensity, i.e. $h=k \sigma$.

$$
Z(i, j)=\sum_{j} e^{-\frac{\left\|g\left(N_{j}\right)-g\left(N_{i}\right)\right\|_{2}^{2}}{h^{2}}}
$$

In our proposed algorithm, we have quantify the perceptual similarity between the underlying patches by incorporating the GMSD metric as a weighting factor along with Euclidean distance. Let $X_{1}$ and $X_{2}$ are the corresponding patches, the GMSD coefficient between them can be mathematically defined as,

$$
G M S D(i)=\sqrt{\frac{1}{N} \sum_{i=1}^{N}(G M S(i)-G M S M)^{2}}
$$

where, GMS is the gradient magnitude similarity map as defined in equation 5. GMSM, gradient magnitude similarity mean, score represents the average of the GMS map. Higher GMSM score reflects higher correlation between the corresponding patches. Also, $c$ in equation 4 is a constant used for numerical stabilization. Furthermore, algorithmic details, working principle and complete analysis of GMSD algorithm can be found in [5].

$$
G M S(i)=\frac{2 g\left(N_{i}\right) g\left(N_{j}\right)+c}{g\left(N_{i}\right)^{2}+g\left(N_{j}\right)^{2}+c}
$$

As in [4], we have also carried out an empirical study to observe the impact of our proposed scheme of incorporating GMSD in the NLM framework. Weighted averages for both the NLM and GMSD-based NLM algorithms were calculated using original image patches. Further, the denoising results are presented in II and are discussed in more detail in the next section. The interesting observation is, in terms of three performance criterion, i.e. PSNR, SSIM and GMSD, considerable improvement in the image quality and gains were observed with the use of GMSD in the NLM framework. However, it is worth mentioning that, practically, we don't have original image patches as we only have the received noisy image available to us. Moreover, this part of the study was done only to demonstrate the usefulness and effectiveness of the proposed scheme over the conventional NLM algorithm.

As we know, that in reality, we have to deal with a noisy version of these original image patches for estimation of similarity and weighted averages. However, only working with an MSE-based metric at higher noise-levels could cause false detections, especially when an image structure succumbs to noise. Such incidents could result in averaging of several pixel values that do not truly belong to the same underlying structure, creating an over smoothing sometimes referred to as patch jittering blur effect (PJBE).

\section{B. Proposed Methodology}

Let $X$ and $Y$ are the original and noisy images, respectively. Whereas, $N$ represents identical and independently distributed Gaussian noise with a variance of $\sigma_{n}^{2}$. Mathematically,

$$
Y=X+N
$$

Lets consider $Y_{1}$ and $Y_{2}$ as the two noisy patches for which the similarity estimates are to be found. Mathematically, they can be defined as,

$$
Y_{1}=X_{1}+N_{1} \quad Y_{2}=X_{2}+N_{1}
$$

where $X_{1}$ and $X_{2}$ as well as $N_{1}$ and $N_{2}$ are uncorrelated with each other. Now here we look to calculate GMSD coefficient to estimate similarity and weighted averages using noisy image patches available to us. As we understand from [5], that GMSD is robust to noise, but even then we first refine the noisy patches by locally estimating the noise using a method proposed in [6]. This technique serves as our first step before we feed the two refined noisy patches to the next stage to calculate GMSD and ED coefficients for similarity estimation and weighting. Authors in [3] have indicated that 


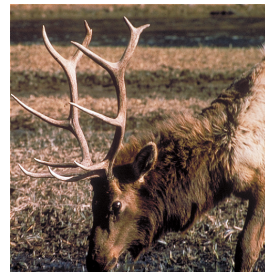

(a) Pristine Img 1

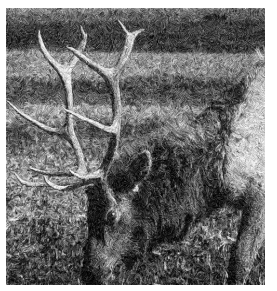

(g) NLM of (b)

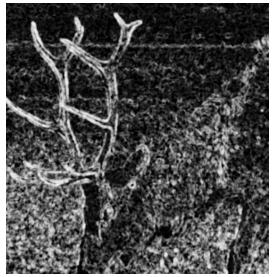

(m) SSIM-QM of (g)

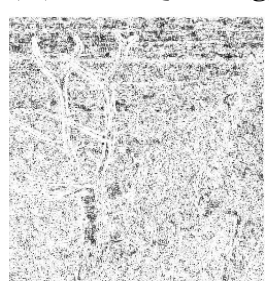

(s) GMS-QM of (g)

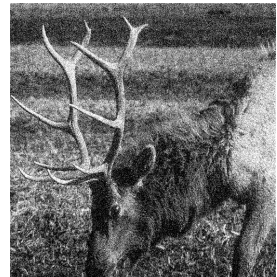

(b) $\mathrm{N}-\mathrm{I} 1 \rightarrow \sigma=\mathbf{0 . 3}$

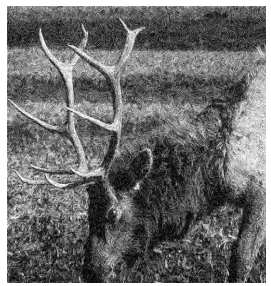

(h) G-NLM of (b)

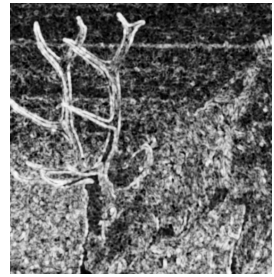

(n) SSIM-QM of (h)

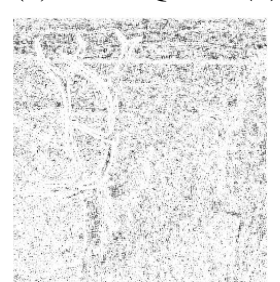

(t) GMS-QM of (h)

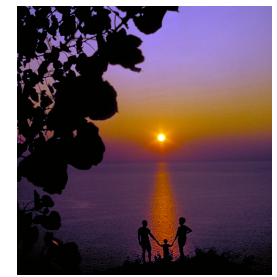

(c) Pristine Img. 2

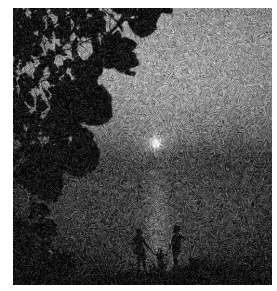

(i) NLM of (d)

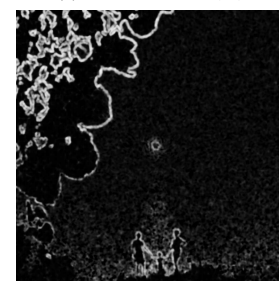

(o) SSIM-QM of (i)

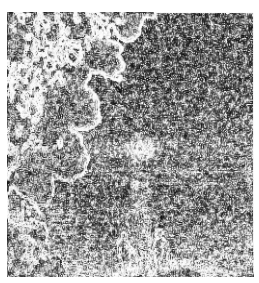

(u) GMS-QM of (i)

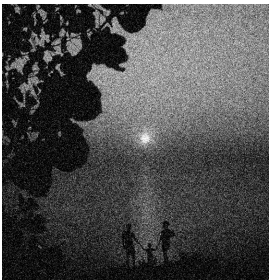

(d) $\mathrm{N}-\mathrm{I} 2 \rightarrow \sigma=\mathbf{0 . 3}$

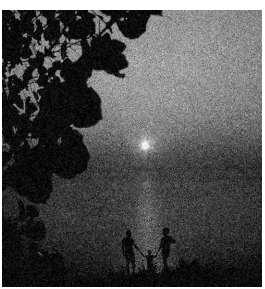

(j) G-NLM of (d)

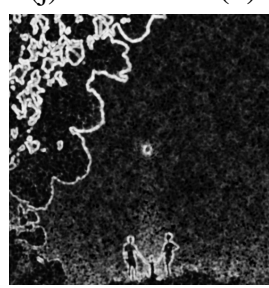

(p) SSIM-QM of (j)

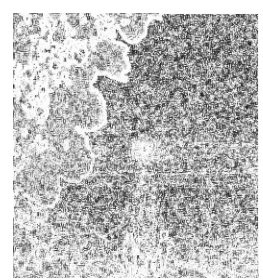

(v) GMS-QM of (j)

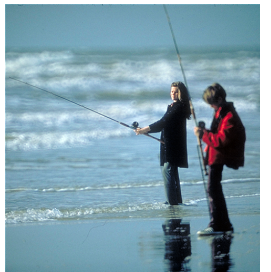

(e) Pristine Img. 3

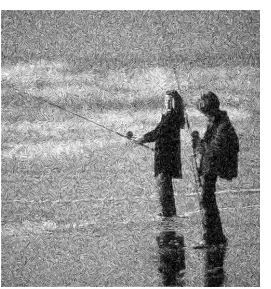

(k) NLM of (f)

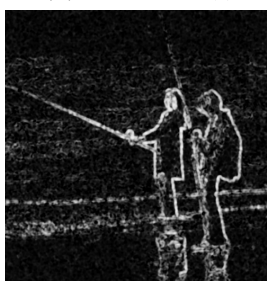

(q) SSIM-QM of (k)

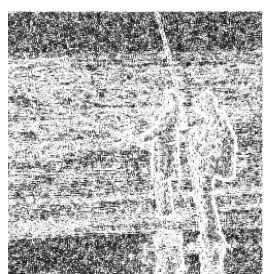

(w) GMS-QM of (k)

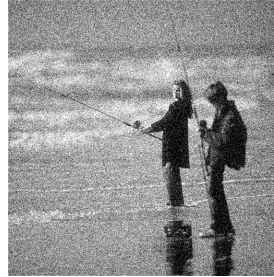

(f) $\mathrm{N}-\mathrm{I} 3 \rightarrow \sigma=\mathbf{0 . 3}$

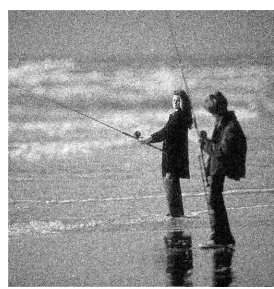

(1) G-NLM of (f)

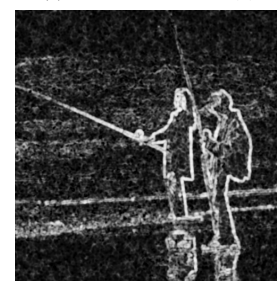

(r) SSIM-QM of (l)

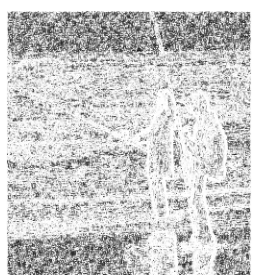

(x) GMS-QM of (l)

Fig. 1: Visual comparison of NLM and GMSD-based-NLM algorithm in terms of GMS and SSIM quality maps. Brighter map indicates better quality.

NLM denoising is based on "method noise" as the residual image obtained after subtracting denoised image from the noise-free image looks like some random noise that does not seem to have structures in it. Therefore, NLM can be useful in estimating local noise for respective patches i.e. $\hat{N}_{1}$ and $\hat{N}_{2}$. Moreover, it helps to remove random noise and give a much-refined version of image patches before actual denoising operation is performed. Mathematically,

$$
\hat{X}_{1}=Y_{1}+\hat{N}_{1} \quad \hat{X}_{1}=Y_{1}+\hat{N}_{1}
$$

After refinement of noisy image patches, the proposed GMSD and ED-based operation for similarity and weight estimation will be made. Mathematically, it is defined as,

$$
\omega(i, j)=\frac{1}{Z(i)} e^{-\frac{\operatorname{GMSD}\left(\hat{X}_{i}, X_{j}\right) \cdot\left\|X_{i}-X_{j}\right\|_{2}^{2}}{h^{2}}}
$$

where,

$$
Z(i)=\sum_{j} e^{-\frac{G M S D\left(\hat{x}_{i}, \hat{X}_{j}\right) \cdot\left\|\hat{X}_{i}-\hat{X}_{j}\right\|_{2}^{2}}{h^{2}}}
$$

where, $w(i, j)$ is the GMSD- and ED-based weighting factor between the two noisy image patches centred at locations $i$ and $j$. After weighted averaging at each pixel location, the final denoised image will be obtained. Mathematically, it is defined as,

$$
U_{i}^{(G M S D-N L)}=\sum_{j \in \Omega} \omega(i, j) \hat{X}_{j}
$$

\section{EXPERIMENTAL DETAILS}

Quality assessment (QA) research depends upon calibrated data as well as good testing mechanisms to make predictions consistent with human observers. Subjective experiments are conducted in a controlled environment to develop databases for QA research. In this section, experimental results for the proposed algorithm on a publicly available subjective database, i.e. Computational and Subjective Image Quality (CSIQ) Image Quality Database [7], have been presented. Moreover, the proposed scheme was tested with STOA image quality measures, i.e. SSIM and GMSD, to estimate the visual quality of the denoised images. 


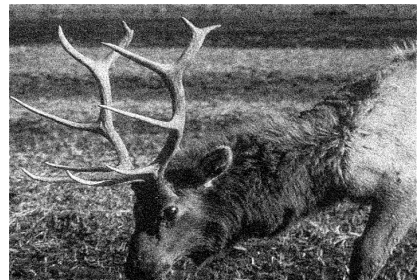

(a) Noisv Image $\rightarrow \sigma=\mathbf{0 . 2}$

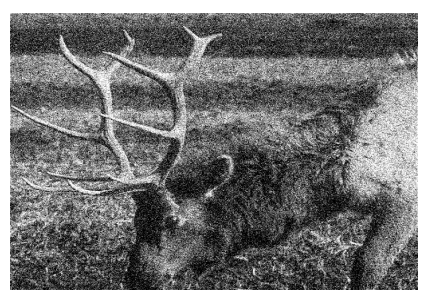

(e) Noisv Image $\rightarrow \sigma=\mathbf{0 . 4}$

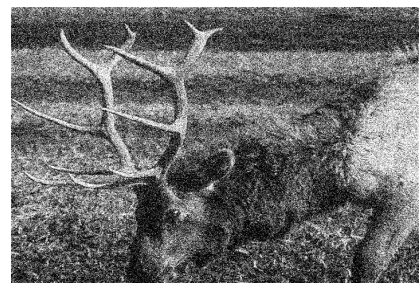

(i) Noisv Image $\rightarrow \sigma=\mathbf{0 . 6}$

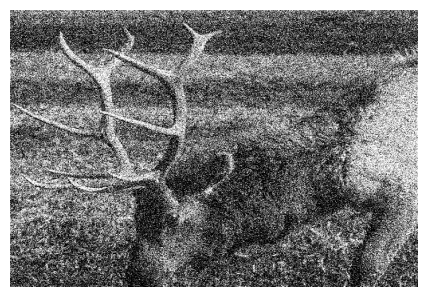

(m) Noisv Image $\rightarrow \sigma=\mathbf{0 . 8}$

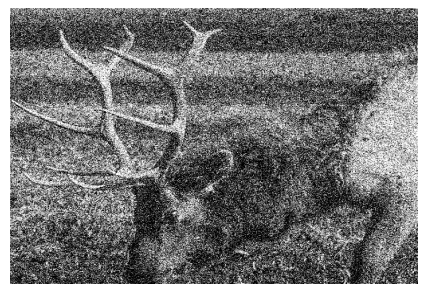

(q) Noisy Image $\rightarrow \sigma=\mathbf{1 . 0}$

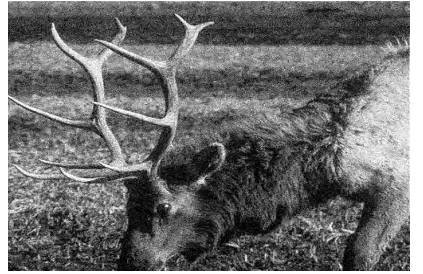

(b) GMSD-NLM Filt. Image

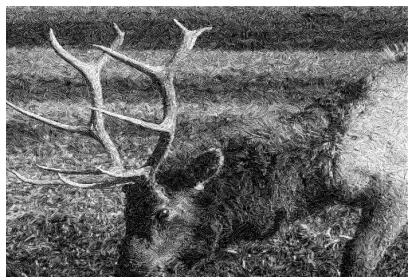

(f) GMSD-NLM Filt. Image

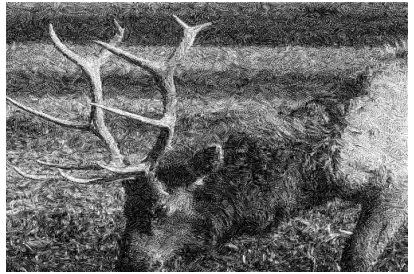

(i) GMSD-NLM Filt. Image

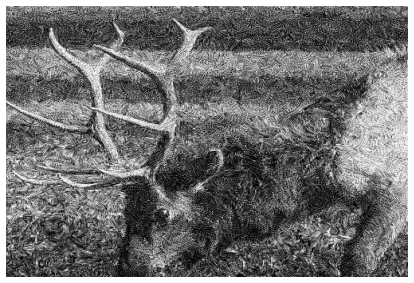

(n) GMSD-NLM Filt. Image

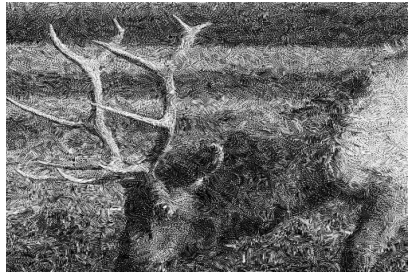

(r) GMSD-NLM Filt. Image

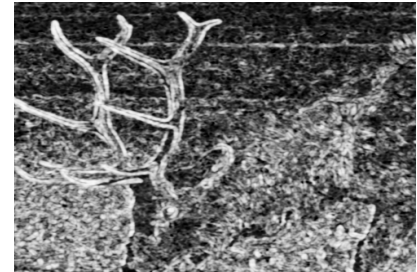

(c) SSIM Map of (b)

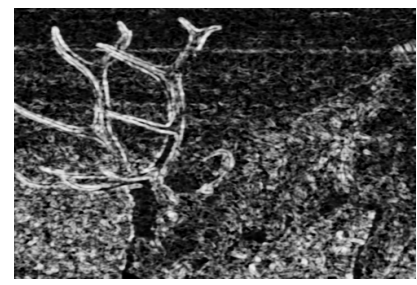

(g) SSIM Map of (f)

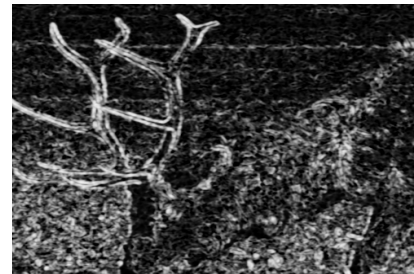

(k) SSIM Map of (j)

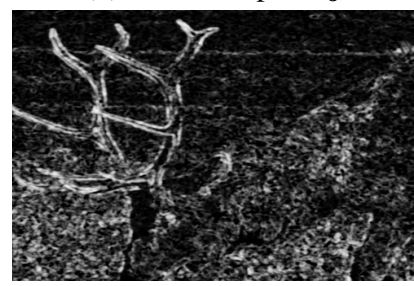

(o) SSIM Map of (n)

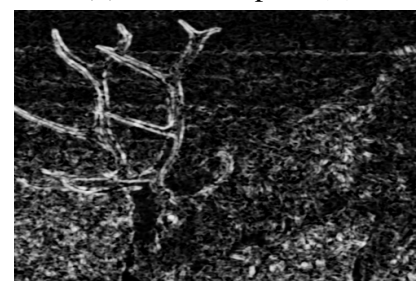

(s) SSIM Map of (r)

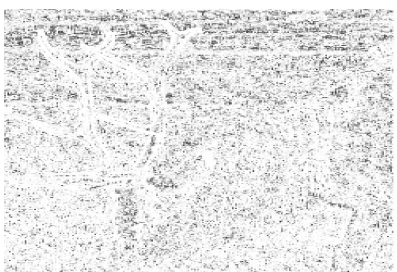

(d) GMS Map of (b)

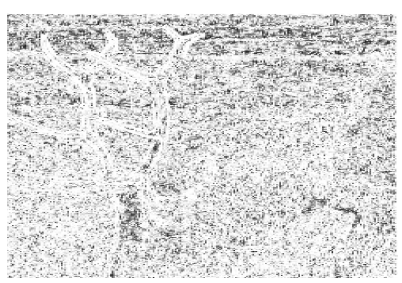

(h) GMS Map of (f)

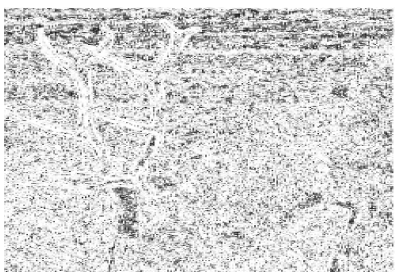

(1) GMS Map of (j)

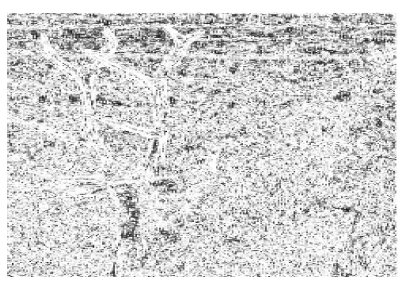

(p) GMS Map of (n)

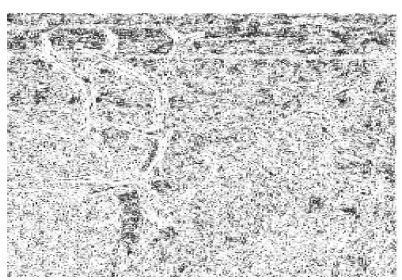

(t) GMS Map of (r)

Fig. 2: Denoising results from the GMSD-based NLM algorithm are depicted on a test image in terms of GMS and SSIM quality maps. The noise variance $\left(\sigma_{\text {normalized }}\right)$ was also varied between 0.1 to 1 with a step of 0.1 .

\section{A. Database and Parametric Details}

The proposed model was validated using the CSIQ database. It is the second-largest subjective database with 30 pristine references and 866 distorted images. We have tested our proposed model using the 30 available references images at different noise levels. However, results on only three reference images are illustrated in figures and tables presented in this paper. Moreover, the identical and independently distributed Gaussian noise was added to each of the pristine images in a controlled environment. The parametric details including, size of similarity window (patch size), size of the search window, filtering parameter (h) and noise variance ( $\sigma$ (normalized)). The parameters are listed in Table I. Moreover, these parametric values were kept the same as the ones used in the original NLM algorithm. The details on the selection of these parameters are found in [8]. Also, as GMSD is part of our proposed scheme, a constant is there in GMSD formulation, that is, $c$; to ensure numerical stability. We set $c=0.0026$, as mentioned in [5]. All simulations presented in this paper have been conducted in MATLAB. 
TABLE I: Parameters for implementation of GMSD-based non-local means filter.

\begin{tabular}{|c|c|c|c|c|}
\hline \multicolumn{5}{|c|}{ For Gray-Scale Images } \\
\hline$\sigma \quad \mid$ & Similarity Window (f) & & Search Window (t) & $\mathrm{h}$ \\
\hline $0.1 \mid$ & $3 \times 3$ & I & $21 \times 21$ & $0.4 \sigma$ \\
\hline 0.21 & $5 \times 5$ & 1 & $21 \times 21$ & $0.4 \sigma$ \\
\hline 0.31 & $5 \times 5$ & 1 & $21 \times 21$ & $0.4 \sigma$ \\
\hline 0.4 । & $7 \times 7$ & 1 & $35 \times 35$ & $0.35 \sigma$ \\
\hline 0.51 & $9 \times 9$ & 1 & $35 \times 35$ & $0.35 \sigma$ \\
\hline \begin{tabular}{ll|}
$0.6 \quad \mid$ \\
\end{tabular} & $9 \times 9$ & I & $35 \times 35$ & $0.35 \sigma$ \\
\hline 0.7 । & $9 \times 9$ & 1 & $35 \times 35$ & $0.35 \sigma$ \\
\hline $0.8 \mid$ & $11 \times 11$ & 1 & $35 \times 35$ & $0.35 \sigma$ \\
\hline 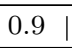 & $11 \times 11$ & I & $35 \times 35$ & $0.35 \sigma$ \\
\hline $1.0 \mid$ & $11 \times 11$ & | & $35 \times 35$ & $0.35 \sigma$ \\
\hline
\end{tabular}

\section{B. Results and Discussion}

Performance evaluation of the proposed model, GMSDbased NLM, has been made using three quality measures; PSNR, SSIM and GMSD. Specifically, the SSIM and GMSD index helps us to quantify as to how close the results are to human visual perception. Better SSIM (near 1) and GMSD (near 0 ) values imply the higher visual quality of images. In Table II and III, the performance of the proposed model is compared with that of the conventional NLM denoising algorithm. It is observed that the proposed scheme outperforms the conventional NLM denoising algorithm by a good margin. It is also observed that not only the visual quality of denoised images get better with the proposed scheme, but the PSNR value has also improved.

Also, in Figure 1 the visual quality of denoised images from both the algorithms are illustrated. It is observed that the SSIM and GMS quality maps for the proposed scheme are brighter than the conventional NLM algorithm, as brighter quality map reflects better visual quality (specific details on the interpretation of quality maps can be found in [5]). In addition, with Figure 2, we have also illustrated quality maps obtained from SSIM and GMSD measures for a denoised test image contaminated at different noise levels (variance) i.e. $\sigma=0.1,0.2,0.3, \ldots, 1.0$. Also, it is an important thing to note that GMSD-based NLM algorithm will always perform better with images that are rich in structures compared to MSE-based conventional NLM algorithm. So, algorithms or metrics with a better understanding of image structures can be instrumental in image reconstruction problems, especially, where the visual quality of images is of prime importance. Though, the addition of an additional metric to the NLM formulation increases the overall computational expense of the system. Nevertheless, it improves the performance of the system by a good margin in terms of the visual quality of the denoised images. To cater to computational expense, researchers have moved towards optimization and machine learning paradigms, especially, deep learning. An example is as described in [9], where denoising problem has been modelled using deep neural networks with good effect. However, visual perception-based reconstruction is still a complicated task and hasn't been catered with much success yet.

\section{CONCLUSION}

In this work, a visual quality assessment metric-based image denoising algorithm has been proposed, that is, gradient magnitude similarity deviation (GMSD)-based non-local means (NLM) filter. We have demonstrated the usefulness and effectiveness of GMSD, a visual quality assessment metric, in capturing local structural variations between corresponding image patches for effective denoising. As we understand, that each local patch in an image has diverse structural features. Once this diversity is captured, it is easier to identify patches with similar characteristics. Therefore, keeping the aforementioned in consideration, GMSD was incorporated in the conventional MSE-based non-local means filter. Moreover, as we know that MSE only deals with visible errors and does not correlate well with human visual perception, so in most cases, usually, the images processed with MSE-based mechanisms have shown poor visual quality, especially, when the noise levels (variance) are high. Simulation results based on PNSR, SSIM and GMSD coefficients have shown that the

TABLE II: Performance comparison of NLM and GMSD-based NLM denoising algorithms while using original-image patches for weight calculations.

\begin{tabular}{|c|c|c|c|c|c|c|c|c|c|c|c|c|c|c|c|c|c|c|c|c|c|c|c|c|c|c|c|c|c|c|}
\hline \multirow{2}{*}{$\begin{array}{l}\text { Test Images } \\
\text { Noise }(\sigma)\end{array}$} & \multicolumn{10}{|c|}{ Image 1} & \multicolumn{10}{|c|}{ Image 2} & \multicolumn{10}{|c|}{ Image 3} \\
\hline & 0.1 & 0.2 & 0.3 & 0.4 & 0.5 & 0.6 & 0.7 & 0.8 & 0.9 & 1.0 & 0.1 & 0.2 & 0.3 & 0.4 & 0.5 & 0.6 & 0.7 & 0.8 & 0.9 & 1.0 & 0.1 & 0.2 & 0.3 & 0.4 & 0.5 & 0.6 & 0.7 & 0.8 & 0.9 & 1.0 \\
\hline \multicolumn{31}{|c|}{ Peak Signal-to-Noise Ratio (PSNR) dB } \\
\hline Noisy Image & $\mid 15.6875$ & 15.2310 & 14.2518 & 14.1121 & 14.0523 & 14.0133 & 13.5033 & 13.2123 & 12.8978 & 12.5687 & | 14.99871 & 14.7832 & 14.7591 & 14.2583 & 13.9896 & 13.5867 & 13.2523 & 12.9985 & 12.7848 & 12.5748 & | 14.9754 & 14.5642 & 14.0420 & 13.8961 & 13.5442 & 13.1625 & 13.0523 & 12.9899 & 12.7845 & 12.5462 \\
\hline NLM & 17.1847 & 16.8457 & 16.5474 & 16.3421 & 15.9582 & 15.7741 & 15.2101 & 14.7212 & 14.3578 & 14.0341 & 17.5784 & 17.2145 & 16.8224 & 16.6101 & 15.9989 & 15.6021 & 14.8214 & 14.5648 & 14.1278 & 13.8565 & 16.7210 & 16.5244 & 16.1377 & 15.9543 & 15.5486 & 15.1243 & 14.9586 & 14.2315 & 14.0155 & 13.8947 \\
\hline GMSD-NLM & 18.8999 & 18.2965 & 17.8545 & 17.5421 & 17.1864 & 16.8951 & 16.5511 & 16.3545 & 15.8678 & 15.6956 & 18.7826 & 18.3851 & 17.9878 & 17.5741 & 16.8967 & 16.5674 & 15.9978 & 15.8789 & 15.5240 & 15.1542 & 18.5475 & 18.1025 & 17.5245 & 17.3564 & 16.9825 & 16.6547 & 16.0654 & 15.7415 & 15.2342 & 14.9992 \\
\hline \multicolumn{31}{|c|}{ Structural Similarity Index (SSIM) } \\
\hline Noisy Image & 0.7546 & 0.7120 & 0.6214 & 0.6012 & 0.5412 & 0.5105 & 0.4023 & 0.3024 & 0.2108 & 0.1105 & 0.7890 & 0.7512 & 0.6942 & 0.6213 & 0.5954 & 0.5302 & 0.4512 & 0.3302 & 0.2204 & 0.1890 & 0.7124 & 0.6952 & 0.6615 & 0.6345 & 0.5124 & 0.4213 & 0.3502 & 0.2503 & 0.1870 & 0.0956 \\
\hline NLM & 0.7905 & 0.7584 & 0.7341 & 0.6589 & 0.6214 & 0.5799 & 0.4954 & 0.4214 & 0.3593 & 0.2214 & 0.8101 & 0.7765 & 0.7389 & 0.6789 & 0.6352 & 0.6012 & 0.5212 & 0.4687 & 0.3641 & 0.2987 & 0.7821 & 0.7364 & 0.6999 & 0.6950 & 0.6106 & 0.5748 & 0.4399 & 0.3341 & 0.2612 & 0.1685 \\
\hline GMSD-NLM & 0.8345 & 0.7857 & 0.7421 & 0.6965 & 0.6357 & 0.6015 & 0.5845 & 0.5564 & 0.4815 & 0.3952 & 0.8897 & 0.8654 & 0.7987 & 0.7548 & 0.7021 & 0.6589 & 0.6235 & 0.5986 & 0.5021 & 0.4012 & 0.8125 & 0.7978 & 0.7545 & 0.7127 & 0.7025 & 0.6654 & 0.5987 & 0.5234 & 0.4518 & 0.3987 \\
\hline \multicolumn{31}{|c|}{ Gradient Magnitude Similarity Deviation (GMSD) } \\
\hline Noisy Image & 0.0112 & 0.0393 & 0.0648 & 0.1387 & 0.1882 & 0.2345 & 0.2545 & 0.2897 & 0.3241 & 0.4052 & 0.0011 & 0.0578 & 0.0989 & 0.1478 & 0.1945 & 0.2154 & 0.2521 & 0.3012 & 0.3781 & 0.4268 & $\mid 0.0035$ & 0.0822 & 0.1011 & 0.1523 & 0.1975 & 0.2357 & 0.2963 & 0.3584 & 0.4150 & 0.4587 \\
\hline NLM & 0.0110 & 0.0212 & 0.0513 & 0.0945 & 0.1610 & 0.2001 & 0.2189 & 0.2625 & 0.2765 & 0.3312 & 0.0010 & 0.0410 & 0.0801 & 0.1199 & 0.1698 & 0.2001 & 0.2299 & 0.2765 & 0.3545 & 0.3865 & 0.0025 & 0.0601 & 0.0902 & 0.1299 & 0.1684 & 0.2089 & 0.2810 & 0.3425 & 0.3865 & 0.4395 \\
\hline GMSD-NLM & \begin{tabular}{|l|l|}
0.0009 \\
\end{tabular} & 0.0165 & 0.0305 & 0.0815 & 0.1387 & 0.1748 & 0.1847 & 0.2102 & 0.2414 & 0.2897 & 0.0009 & 0.0350 & 0.0721 & 0.1084 & 0.1352 & 0.1599 & 0.1845 & 0.2237 & 0.2861 & 0.3128 & 0.0011 & 0.0489 & 0.0654 & 0.0995 & 0.1125 & 0.1546 & 0.2214 & 0.2514 & 0.3012 & 0.3512 \\
\hline
\end{tabular}


TABLE III: Performance comparison of NLM and GMSD-based NLM denoising algorithms while using noisy-image patches for weight calculations.

\begin{tabular}{|c|c|c|c|c|c|c|c|c|c|c|c|c|c|c|c|c|c|c|c|c|c|c|c|c|c|c|c|c|c|c|}
\hline Test Images & & & & & & & & & & & & & & & & & & & & & & & & & & & & & & \\
\hline Noise $(\sigma)$ & 0.1 & 0.2 & 0.3 & 0.4 & 0.5 & 0.6 & 0.7 & 0.8 & 0.9 & 1.0 & 0.1 & 0.2 & 0.3 & 0.4 & 0.5 & 0.6 & 0.7 & 0.8 & 0.9 & 1.0 & 0.1 & 0.2 & 0.3 & 0.4 & 0.5 & 0.6 & 0.7 & 0.8 & 0.9 & 1.0 \\
\hline
\end{tabular}

\begin{tabular}{l|llllllllll|lllllllllllllllllllllll}
\hline Noisy Image & 15.6875 & 15.2310 & 14.2518 & 14.1121 & 14.0523 & 14.0133 & 13.5033 & 13.2123 & 12.8978 & 12.5687 & 14.9987 & 14.7832 & 14.7591 & 14.2583 & 13.9896 & 13.5867 & 13.2523 & 12.9985 & 12.7848 & 12.5748 & 14.9754 & 14.5642 & 14.0420 & 13.8961 & 13.5442 & 13.1625 & 13.0523 & 12.9899 & 12.7845 & 12.5462
\end{tabular} \begin{tabular}{l|llllllllllll|lllllllllllllllllllllll} 
NLM & 17.0235 & 16.7532 & 16.3394 & 16.1385 & 15.8985 & 15.7545 & 15.1235 & 14.7135 & 14.3451 & 14.0214 & 17.4215 & 17.2105 & 16.8004 & 16.5421 & 15.9845 & 15.5325 & 14.7214 & 14.5582 & 14.1012 & 13.8565 & 16.7210 & 16.5244 & 16.1377 & 15.9543 & 15.5486 & 15.1243 & 14.9586 & 14.2315 & 14.0155 & 13.8947
\end{tabular}

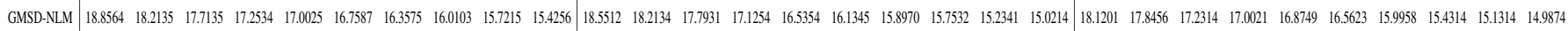

\section{Structural Similarity Index (SSIM)}

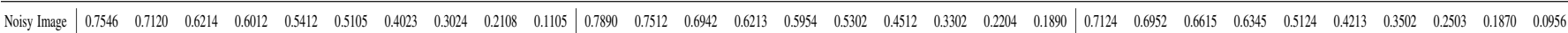

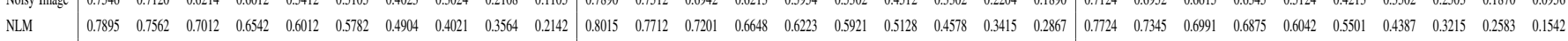

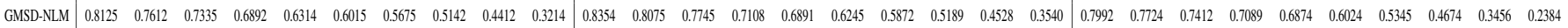
Gradient Magnitude Similarity Deviation (GMSD)

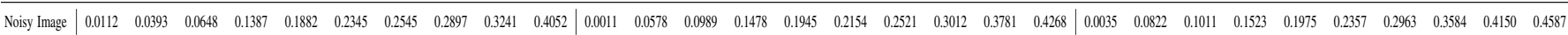
\begin{tabular}{l|llllllllllllllllllllllllllllll} 
NLM & 0.0110 & 0.0215 & 0.0541 & 0.0962 & 0.1645 & 0.2054 & 0.2387 & 0.2745 & 0.2847 & 0.3574 & 0.0010 & 0.0478 & 0.0871 & 0.1254 & 0.1754 & 0.2012 & 0.2347 & 0.2845 & 0.3648 & 0.4010 & 0.0029 & 0.0622 & 0.0924 & 0.1347 & 0.1744 & 0.2145 & 0.2874 & 0.3471 & 0.3901 & 0.4492
\end{tabular}

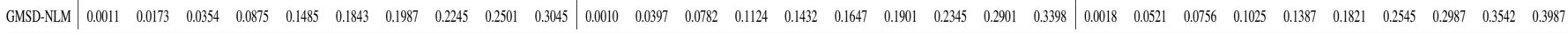

algorithm has promise, robustness and works well even at high noise levels.

\section{REFERENCES}

[1] Z. Wang, A. C. Bovik, H. R. Sheikh, E. P. Simoncelli et al., "Image quality assessment: from error visibility to structural similarity," IEEE transactions on image processing, vol. 13, no. 4, pp. 600-612, 2004.

[2] H. Rajagopal, A. S. M. Khairuddin, N. Mokhtar, A. Ahmad, and R. Yusof, "Application of image quality assessment module to motionblurred wood images for wood species identification system," Wood Science and Technology, vol. 53, no. 4, pp. 967-981, Jul 2019. [Online]. Available: https://doi.org/10.1007/s00226-019-01110-2

[3] A. Buades, B. Coll, and J.-M. Morel, "A non-local algorithm for image denoising," in 2005 IEEE Computer Society Conference on Computer Vision and Pattern Recognition (CVPR'05), vol. 2. IEEE, 2005, pp. 60-65.

[4] A. Rehman and Z. Wang, "Ssim-based non-local means image denoising," in 2011 18th IEEE International Conference on Image Processing. IEEE, 2011, pp. 217-220.
[5] W. Xue, L. Zhang, X. Mou, and A. C. Bovik, "Gradient magnitude similarity deviation: A highly efficient perceptual image quality index," IEEE Transactions on Image Processing, vol. 23, no. 2, pp. 684-695, Feb 2014.

[6] A. Buades, B. Coll, and J.-M. Morel, "A review of image denoising algorithms, with a new one," Multiscale Modeling \& Simulation, vol. 4, no. 2 , pp. 490-530, 2005

[7] E. C. Larson and D. M. Chandler, "Most apparent distortion: fullreference image quality assessment and the role of strategy," Journal of Electronic Imaging, vol. 19, no. 1, p. 011006, 2010.

[8] A. Buades, B. Coll, and J.-M. Morel, "Non-local means denoising," Image Processing On Line, vol. 1, pp. 208-212, 2011.

[9] C. Tian, Y. Xu, L. Fei, and K. Yan, "Deep learning for image denoising: A survey," CoRR, vol. abs/1810.05052, 2018. [Online]. Available: http://arxiv.org/abs/1810.05052 